Supporting Information

\title{
Porous Fibers Templated by Melt Blowing Co-Continuous Immiscible Polymer Blends
}

Aditya Banerji, ${ }^{\dagger}$ Kailong Jin, ${ }^{\dagger} \S$ Mahesh K. Mahanthappa,${ }^{\dagger}$ Frank S. Bates,,${ }^{\dagger}$ and Christopher J. Ellison $*, \dagger$

\footnotetext{
†Department of Chemical Engineering and Materials Science, University of Minnesota, Minneapolis, MN 55455, United States

${ }^{\S}$ Department of Chemical Engineering, Arizona State University, Tempe, AZ 85287 USA

*To whom correspondence should be addressed: bates001@umn.edu; cellison@umn.edu
}

\section{Materials and Methods}

Materials. Polystyrene (PS), high density polyethylene (HDPE), and polystyrene-blockpoly(ethylene-ran-butylene)-block-polystyrene (SEBS) were purchased from Sigma-Aldrich and used as received. Sigma-Aldrich product numbers for PS, HDPE, and SEBS used for this study are 430102, 428019, and 200565, respectively. The chemical structures and molecular characteristics are shown in Table 1. Thermal and rheological properties of the polymers are provided in Figure S2. PS had a reported $M_{\mathrm{w}} \sim 192,000 \mathrm{~g} / \mathrm{mol}$, melt flow index of 6-9 g/10 min $\left(200{ }^{\circ} \mathrm{C} / 5 \mathrm{~kg}\right.$ ). HDPE had a reported density of $0.947 \mathrm{~g} / \mathrm{mL}$ at $25^{\circ} \mathrm{C}$, a melt flow index of $42 \mathrm{~g} / 10$ $\min \left(190^{\circ} \mathrm{C} / 2.16 \mathrm{~kg}\right.$ ), and a melting temperature of $\sim 126^{\circ} \mathrm{C}$ (Figure S2). SEBS had a reported $M_{\mathrm{w}}$ $\sim 89,000 \mathrm{~g} / \mathrm{mol}$ and a mole ratio [styrene]:[olefin] $=29: 71$. Tetrahydrofuran $(\geq 99.6 \%$; Fisher $)$ solvent was used as received.

Preparation of polymer blends. PS, HDPE, and SEBS were dried overnight in a vacuum oven at $70{ }^{\circ} \mathrm{C}$ to remove residual moisture prior to melt blending in a recirculating, conical twin-screw microcompounder (DSM Xplore, $5 \mathrm{~mL}$ capacity). Melt blending was performed at $250{ }^{\circ} \mathrm{C}$ for 15 min with $50 \mathrm{rpm}$ screw rotation speed under dry nitrogen purge. Binary 60/40 wt \% PS/HDPE blends containing $0.25,0.5$ and $1 \mathrm{wt} \%$ SEBS were prepared by melt blending appropriate amounts 
of PS, HDPE, and SEBS. The resulting blend was extruded through a $2.5 \mathrm{~mm}$ diameter die and water cooled. For an example, a $4 \mathrm{~g}$ batch of 60/45 wt $\%$ PS/HDPE blend with 1 wt $\%$ SEBS contained $2.4 \mathrm{~g}$ of PS, $1.6 \mathrm{~g}$ of HDPE, and $40 \mathrm{mg}$ of SEBS. The compositions for the PS/HDPE binary blends were chosen to obtain the desired cocontinuous morphology, while the SEBS content was varied to tune the co-continuous domain size and coarsening rate.

Melt blowing. To produce nonwoven fibers, the blends were loaded into a custom-built lab-scale melt blowing apparatus, which contained a homemade melt blowing die and fiber collector fitted to a commercial Goettfert ${ }^{\mathrm{TM}}$ Rheo-Tester 1500 capillary rheometer. (References [1-3] provide more details regarding our lab-scale melt blowing apparatus.) The capillary rheometer was used to heat the polymer to the melt blowing temperature and to extrude the polymer through a singlehole melt blowing orifice die with a diameter of $400 \mu \mathrm{m}$ at a controlled polymer flow rate of 0.8 $\mathrm{g} /(\mathrm{min}$ hole $)$. Melt blowing was carried $\sim 5 \mathrm{~min}$ after the sample reached the melt blowing temperature (required $\sim 5 \mathrm{~min}$ to reach temperature). The heated air flow rate was 5.5 standard cubic feet per minute $(\mathrm{SCFM})$ and the air pressure at the die exit was $\sim 6$ psi. $T_{\text {polymer }}=T_{\text {die }}$ was maintained at the die exit, while $T_{\text {air }}=T_{\text {polymer }}+10{ }^{\circ} \mathrm{C}$ was used throughout the melt blowing process. $T_{\text {polymer }}$ was chosen to be $250{ }^{\circ} \mathrm{C}, 260{ }^{\circ} \mathrm{C}$, and $280{ }^{\circ} \mathrm{C}$ for PS/HDPE, PS/HDPE/0.25 wt \% SEBS and PS/HDPE/1 wt \% SEBS blends, respectively. Melt blown fibers were collected using a stationary collector consisting of a stainless-steel screen. The collector was located $\sim 1 \mathrm{~m}$ from the die orifice and was maintained at ambient temperature.

Selective extraction of blend components. PS was removed from the samples by soaking in tetrahydrofuran at $50{ }^{\circ} \mathrm{C}$ for $4 \mathrm{~h}$ (for bulk blends) and $30 \mathrm{~min}$ (for melt blown fibers embedded in epoxy). Under these conditions, only PS can be dissolved but HDPE cannot. The bulk blend samples were re-soaked in fresh THF for another $4 \mathrm{~h}$. The insoluble fractions were then dried under vacuum at $50{ }^{\circ} \mathrm{C}$ for $12 \mathrm{~h}$. The amount of PS removed was measured by comparing the weights of the sample before and after solvent extraction. 
Differential scanning calorimetry (DSC). Differential scanning calorimetry was performed with a Mettler Toledo DSC 1 calorimetry instrument. Approximately 5-10 mg of a sample was loaded into a hermetically sealed aluminum pan for each DSC run. Materials were heated to $175^{\circ} \mathrm{C}$ for 5 min to erase thermal history, cooled to $25^{\circ} \mathrm{C}$ at $20{ }^{\circ} \mathrm{C} / \mathrm{min}$, and then heated to $200{ }^{\circ} \mathrm{C}$ at $10{ }^{\circ} \mathrm{C} / \mathrm{min}$. Glass transition $\left(T_{\mathrm{g}, 1 / 2 \Delta \mathrm{Cp}}\right)$ and melting temperatures $\left(T_{\mathrm{m}, \text { endothermic peak }}\right)$ were determined from the second heating ramp.

Proton nuclear magnetic resonance $\left({ }^{1} \mathbf{H}\right.$ NMR). Proton nuclear magnetic resonance spectrometry was performed with a $500 \mathrm{MHz}$ Bruker Avance III spectrometer. Melt blown fiber specimens ( 7-8 mg) were dissolved in hot 1,1,2,2-Tetrachloroethane- $\mathrm{d}_{2}\left(\mathrm{Cl}_{2} \mathrm{CDCDCl}_{2}, \mathrm{TCE}-\mathrm{d}_{2}\right)$ $(\sim 0.7 \mathrm{~mL})$ and analyzed using ${ }^{1} \mathrm{H}$ NMR spectroscopy experiments at $100{ }^{\circ} \mathrm{C}$.

Rheological measurements. Rheological properties were measured with a TA Instruments ARES-G2 rheometer equipped with a $25 \mathrm{~mm}$ parallel-plate fixture. All experiments were performed in the linear viscoelastic region, which was determined by dynamic strain sweep tests. Isothermal dynamic frequency sweep measurements were conducted between 0.1 and $100 \mathrm{rad} / \mathrm{s}$ to measure the complex viscosities $\left(\eta^{*}\right)$ at either 250,260 or $280{ }^{\circ} \mathrm{C}$.

\section{Scanning electron microscopy (SEM) and image analyses. (a) Blend morphology} characterization: Polymer blends were freeze-fractured in liquid nitrogen followed by selective extraction of PS. The washed samples were coated with $\sim 7 \mathrm{~nm}$ of iridium with a ACE600 Coater for morphology visualization with a Hitachi S4700 SEM. The procedure for analyzing the cocontinuous domains sizes by image segmentation and measuring the resulting interfacial length from a SEM image is as follows: A representative SEM image shown in Fig S1a is segmented using the Fiji software (an image analysis package based on ImageJ developed by NIH) to yield Fig S1b. The segmented image is then transformed to show the outlines of the cocontinuous domains as shown in Fig S1c. The summation of the perimeter of the domains provided the total interfacial length in the selected SEM image. Using the area of the calibrated SEM image, the characteristic domain size was calculated using Equation 2 from the main text. The reported error 
(a)

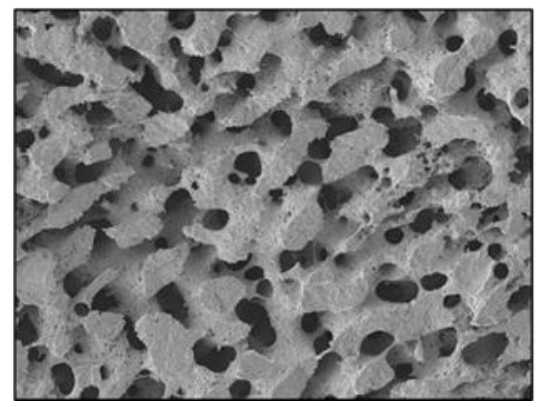

(b)

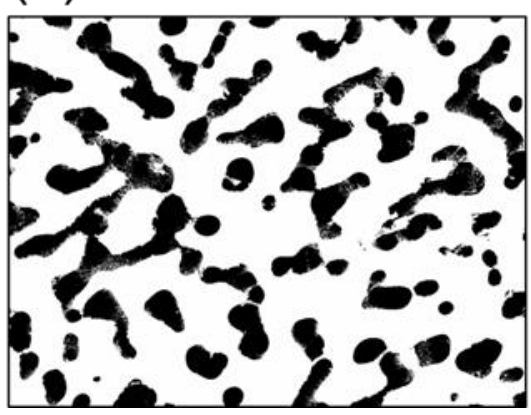

(c)

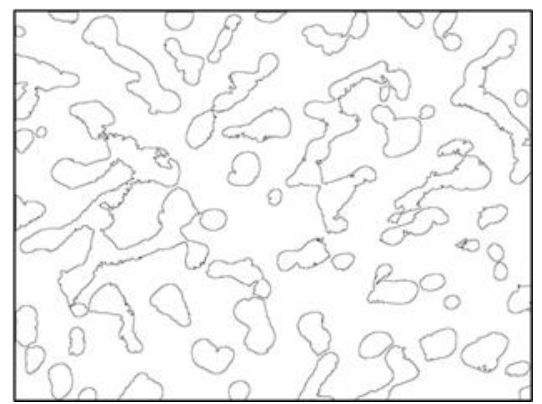

Figure S1: (a) A representative SEM image showing the cocontinuous morphology; (b) A binary image obtained by image segmentation of the SEM image using Fiji image analysis package; (c) Outlines of the domains obtained after image segmentation

bars were the standard deviations calculated from at least three independent image analysis measurements. (b) Fiber mat imaging: Melt blown fiber samples were coated with $\sim 7 \mathrm{~nm}$ layer of iridium using an ACE600 Coater. For each fiber mat, 5-8 SEM micrographs were taken and at least 100 fiber diameter measurements were made using Fiji software. Then, a log-normal distribution function (Equation S1) was fit to the fiber diameter data. ${ }^{2}$ The average $\left(d_{\text {avg }}\right)$ and standard deviation $(\sigma)$ of the diameter distribution of the fiber mats obtained at different conditions were extracted from the corresponding normal fits.

$$
f(d)=\frac{1}{d \sigma \sqrt{2 \pi}} \exp \left[-\frac{1}{2 \sigma^{2}}\left\{\ln \left(\frac{d}{d_{\text {avg }}}\right)\right\}^{2}\right]
$$

(c) Fiber cross-section morphology characterization: The melt blown fibers were first embedded in an epoxy (Poly/Bed 812) matrix, followed by freeze-fracturing in liquid nitrogen and selective extraction of PS. Multiple SEM images of the fiber cross-sections were analyzed using the Fiji image analysis package to calculate the arithmetic mean pore sizes by measuring at least 300 pores across fiber cross-sections. 


\section{Additional Characterization and Results}
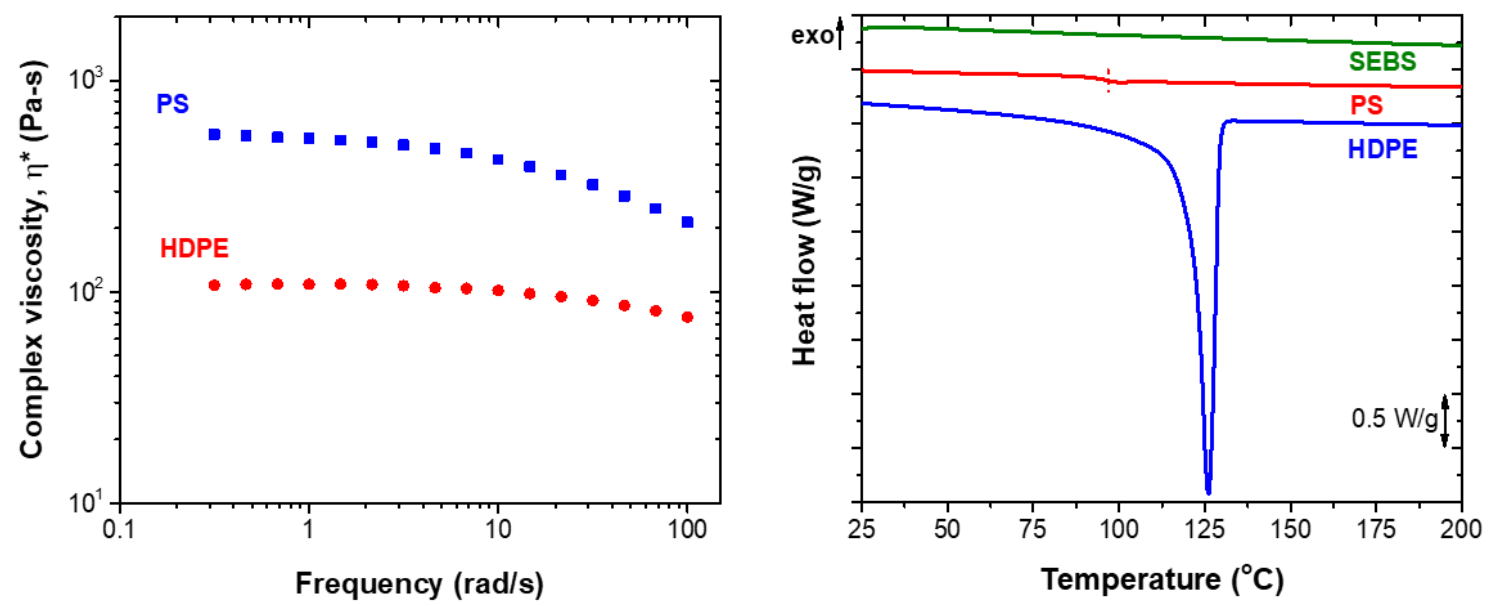

Figure S2: (Left) $\eta^{*}$ versus frequency for neat PS and HDPE at $250{ }^{\circ} \mathrm{C}$. All rheological measurements were performed in the linear viscoelastic region. (Right) DSC thermograms of neat SEBS, PS, and HDPE. All thermograms were obtained from the second heating ramp at $10{ }^{\circ} \mathrm{C} / \mathrm{min}$ after annealing at $175^{\circ} \mathrm{C}$ for $5 \mathrm{~min}$, followed by quenching to $25^{\circ} \mathrm{C}$ at $20{ }^{\circ} \mathrm{C} / \mathrm{min}$. 


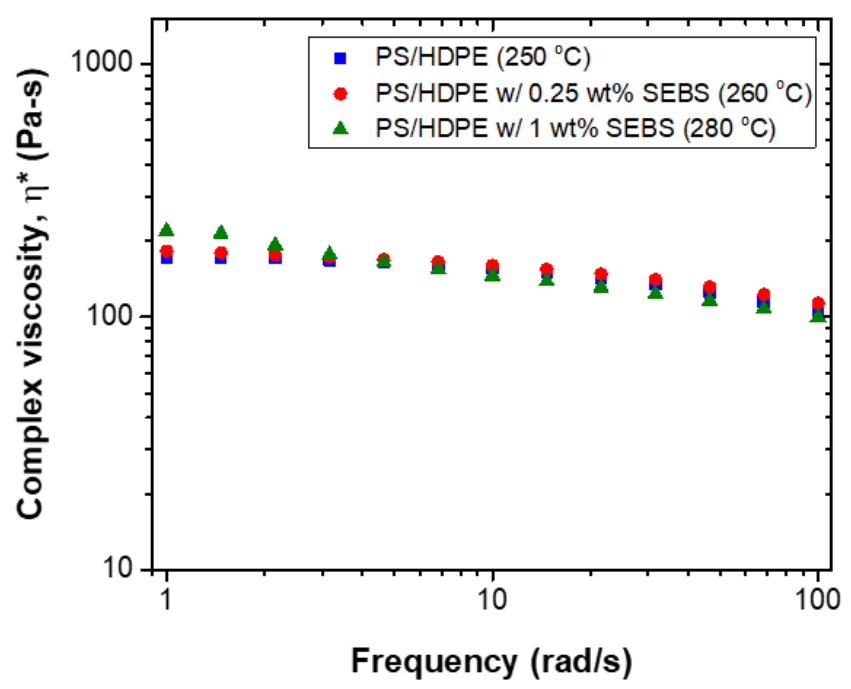

Figure S3: $\eta *$ versus frequency for three blends: 60/40 wt $\%$ PS/HDPE, 60/40/0.25 wt\% PS/HDPE/SEBS, and 60/40/1 wt\% PS/HDPE/SEBS measured at $250{ }^{\circ} \mathrm{C}, 260{ }^{\circ} \mathrm{C}$, and $280{ }^{\circ} \mathrm{C}$, respectively. All rheological measurements were performed in the linear viscoelastic regime. 
60/40 PS/HDPE

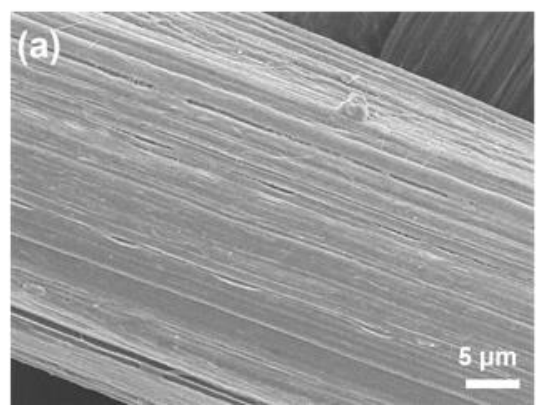

60/40/0.25 wt \% PS/HDPE/SEBS

\section{(b)}

$5 \mu \mathrm{m}$
60/40/1 wt \% PS/HDPE/SEBS

(c)

Figure S4: Representative SEM images of the surfaces of porous melt blown fiber mats obtained from (a) 60/40 wt\% PS/HDPE, (b) 60/40/0.25 wt\% PS/HDPE/SEBS, and (c) 60/40/1 wt\% PS/HDPE/SEBS. All the fiber specimens were imaged after selective extraction of PS.

(a)

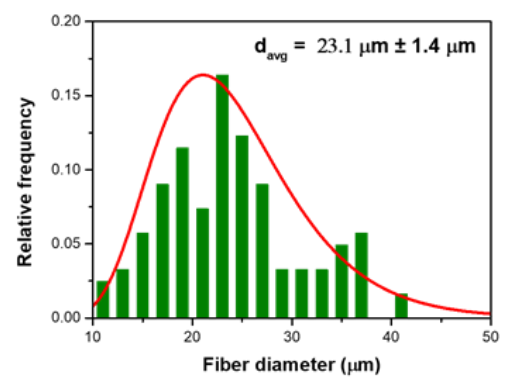

(b)

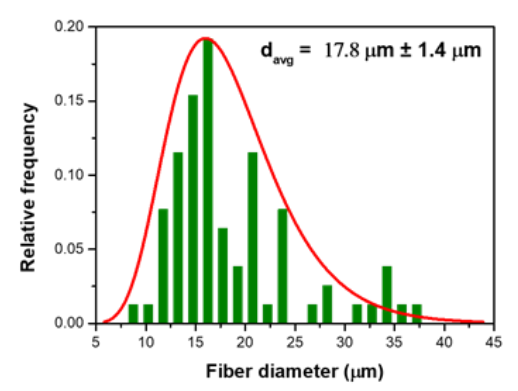

(c)

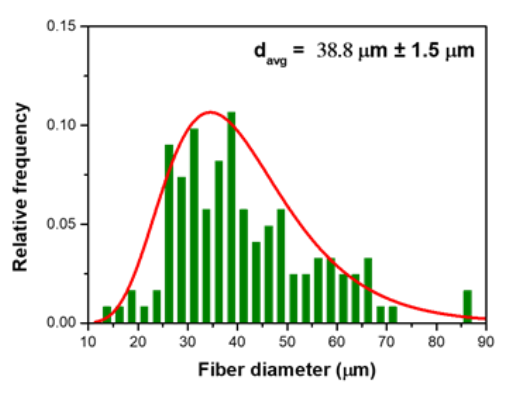

Figure S5: Statistical analyses of the melt blown fiber diameter obtained from (a) 60/40 wt $\%$ PS/HDPE, (b) 60/40/0.25 wt\% PS/HDPE/SEBS, and (c) 60/40/1 wt\% PS/HDPE/SEBS. All the fiber specimens were imaged and analyzed after selective extraction of PS. Two-sample ttests performed between the three sets of fiber diameter data resulted in p-values very close to zero, indicating that the individual datasets are significantly different than each other. 
60/40/0.25 wt \% PS/HDPE/SEBS

60/40/1 wt \% PS/HDPE/SEBS
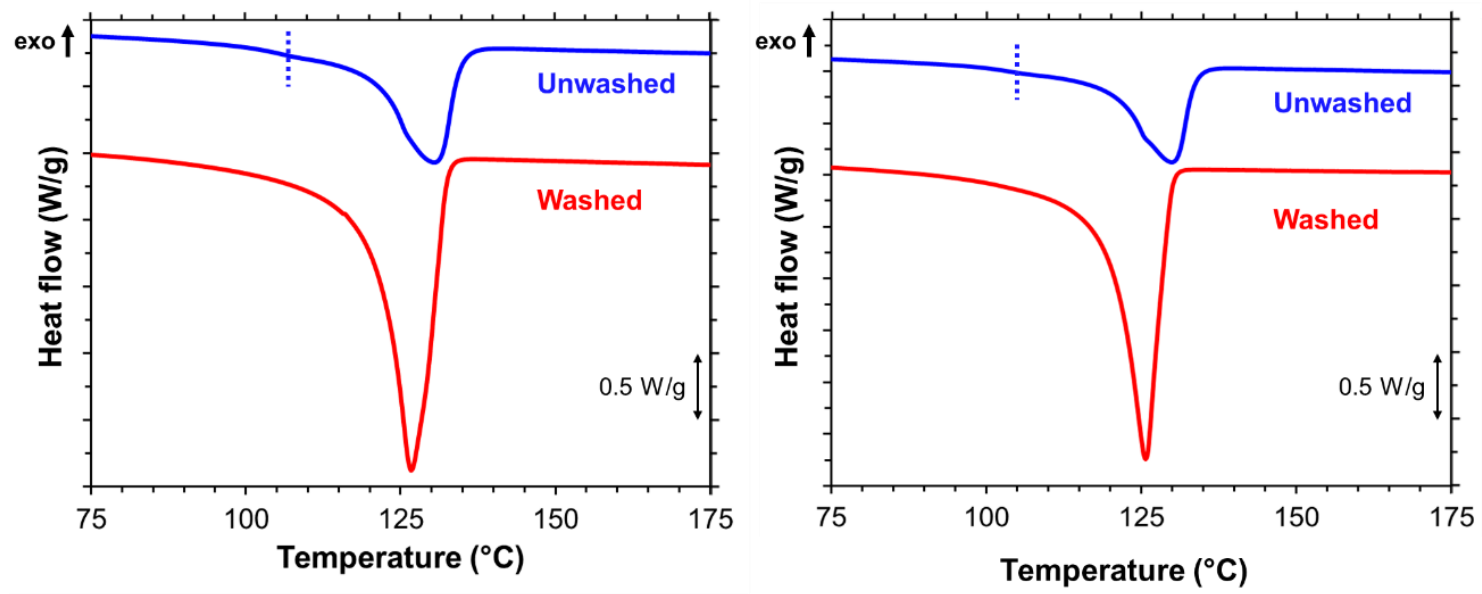

Figure S6: DSC thermograms of melt blown fiber mats obtained from (left) 60/40/0.25 wt \% PS/HDPE/SEBS, and (right) 60/40/1 wt \% PS/HDPE/SEBS, before and after soaking in THF. All thermograms were obtained from the second heating ramp at $10{ }^{\circ} \mathrm{C} / \mathrm{min}$ after annealing at $175{ }^{\circ} \mathrm{C}$ for $5 \mathrm{~min}$, followed by quenching to $25^{\circ} \mathrm{C}$ at $20^{\circ} \mathrm{C} / \mathrm{min}$.
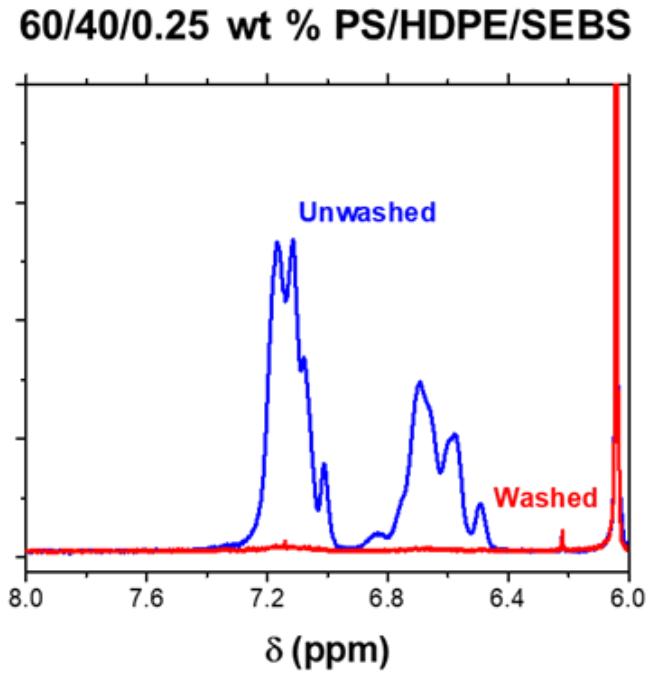

60/40/1 wt \% PS/HDPE/SEBS

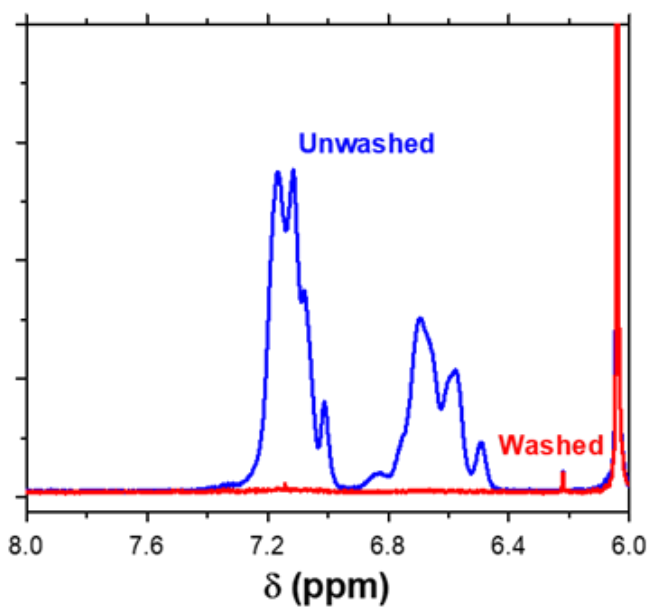

Figure S7: ${ }^{1} \mathrm{H}$ NMR spectra of melt blown fiber mats obtained from (left) $60 / 40 / 0.25$ wt $\%$ PS/HDPE/SEBS, and (right) 60/40/1 wt \% PS/HDPE/SEBS, before and after soaking in THF. All fiber specimens were dissolved in hot TCE- $\mathrm{d}_{2}$ and ${ }^{1} \mathrm{H}$ NMR experiments were performed at $100{ }^{\circ} \mathrm{C}$. The peak at $\sim 6 \mathrm{ppm}$ corresponds to the TCE- $\mathrm{d}_{2}$ solvent. 


\section{References}

1. Zuo, F.; Tan, D. H.; Wang, Z.; Jeung, S.; Macosko, C. W.; Bates, F. S., Nanofibers from Melt Blown Fiber-in-Fiber Polymer Blends. ACS Macro Lett. 2013, 2, 301-305.

2. Ellison, C. J.; Phatak, A.; Giles, D. W.; Macosko, C. W.; Bates, F. S., Melt Blown Nanofibers: Fiber Diameter Distributions and Onset of Fiber Breakup. Polymer 2007, 48, 3306-3316.

3. Tan, D. H.; Zhou, C.; Ellison, C. J.; Kumar, S.; Macosko, C. W.; Bates, F. S., Meltblown Fibers: Influence of Viscosity and Elasticity on Diameter Distribution. J. Non-Newton. Fluid Mech. 2010, 165, 892-900. 